\title{
Multiple endocrine neoplasia and primary hyperparathyroidism - practical approach
}

\author{
Jarosław Koza \\ Chair of Gastroenterology and Nutrition Disorders, Faculty of Health Sciences, Nicolaus Copernicus University in Toruń, \\ Collegium Medicum, Bydgoszcz, Poland
}

Contemp Oncol (Pozn) 2015; 19 (4): 343-344 DOI: $10.5114 /$ wo.2015.54392

\section{The main issue}

Neuroendocrine tumours can be associated with genetic syndromes [1] and this fact should influence the medical procedures. In my work as a physician I met several patients with cancer in familiar history (e.g. colon cancer in the mother and grandmother) and despite the recommendations they avoid screening for the disease. In this year I was dealing with an adult male patient suffering because of a neuroendocrine tumour affecting duodenum with metastases to the liver. In 2013 due to the diagnosis of low-energy fractures, the hyperparathyroidism had been diagnosed in this man. The patient on account of the well-being neglected treatment and doctor visits. Since the origin of 2015 year he began to feel worse. He felt weakness and complained a loss of body weight despite the steady food supply.

Above introduction concerning the patient from my practice prompted me to reflect on the relationship of individual components of familial syndromes associated with neuroendocrine tumours. Although the primary hyperparathyroidism usually originates from benign adenoma without any relationship to syndromes associated with endocrine tumours, in some cases it can develop from the existing multiple endocrine neoplasia type 1 and $2 a$ (MEN 1 and MEN 2 a respectively) as well as be the result of hereditary hyperparathyroidism jaw tumour syndrome [1, 2]. There are also authors (e.g. Thakker, 2014) using names MEN2 for MEN2A, MEN3 for MEN2B and distinguish the type MEN4 for some form classified until recently to MEN1, but with a different genetic mutation. Abnormalities of CDKN1B gene which in man is located on chromosome 12p13 are considered to be the cause of MEN4. Parathyroid adenoma, pituitary adenoma, reproduction organ tumours (e.g. testicular cancer, neuroendocrine cervical carcinoma), adrenal and renal tumours are classified as components of MEN4 syndrome [3]. There are no reports of any others familiar syndromes associated with neuroendocrine tumours and primary hyperparathyroidism. From written previously syndromes causing primary hyperparathyroidism MEN1 is the most frequent and the best known. Primary hyperparathyroidism is usually the first in medical history and the most common endocrynopathies in MEN1
[2]. Although incidence of MEN1 in patients diagnosed with primary hyperparathyroidism is estimated in range of $2-4 \%$, the hyperparathyroidism reaches nearly $100 \%$ penetrance by the age of 50 years in MEN1 patients [2]. The others syndromes associated with neuroendocrine tumours carry even lower probability of primary hyperparathyroidism e.g. in MEN2/MEN2a it is rarer. It occurs in $20-30 \%$ of patients, is also later in natural history and clinical course of hyperparathyroidism is milder than in MEN1 [1, 4].

Certainly, it can be assumed that a neuroendocrine tumour diagnosed two years earlier (for example with the screening tests of chromogranine A and further endoscopic or imaging diagnostics in case of a positive test of chromogranine) would improve the prognosis of described previously patient [5]. Guidelines recommend suspicion of MEN1 in case of primary hyperparathyroidism caused by multiglandular hyperplasia and/or adenoma, or recurrent primary hyperparathyroidism, and additionally the hyperparathyroidism must be accompanied by the one or more features of: duodenal and/or pancreatic endocrine tumours, gastric enterochromaffin - like tumours, anterior pituitary adenoma, adrenocortical tumours or foregut carcinoid tumours [1]. Moreover MEN-1 should be considered as cause of primary hyperparathyroidism when occurred below the age of 30 years [6].

Primary hyperparathyroidism can be manifested by a variety of symptoms from many organs and systems, e.g. from: musculoskeletal system (bone pain, fractures, muscle weakness), kidney (pain due to stone disease, polyuria due to nephrogenic diabetes insipidus or diabetes mellitus), gastrointestinal system (abdominal pain due to peptic ulcer disease or pancreatitis, constipation), cardiovascular system (hypertension, arrhythmia) as well as depression [2]. Although symptomatology is rich, primary hyperparathyroidism can be asymptomatic or the only one symptom can be found. Previously described patient because of poor symptomatology had completely neglected the treatment as well as supplementary diagnostic regarding other more dangerous diseases. There are in Poland screening programs in many cases realised with very good effects e.g. concerning the cancer of the liver [7] or 
colorectal cancer $[8,9]$. The greater emphasis should be put on prevention, prophylaxis and detection of diseases in the earliest stages. This statement should also apply in the field of rare diseases, such as multiple endocrine neoplasia. Publications summarizing current knowledge about relationships between particular diseases within familial syndromes should be considered very valuable [e.g. 1, 3].

\section{Conclusion}

Potential possibility of neuroendocrine tumours should always be taken into consideration in the primary hyperparathyroidism and in special cases [1,4] patients should always be referred for further diagnostic tests in order to recognize a possible tumour in the earliest stage. This relationship should function in the other way also, i.e. neuroendocrine tumour detection should prompt further evaluation. The searching for possible coexisting tumours as well as hyperparathyroidism should be also considered.

The author declares no conflict of interest.

\section{References}

1. Gut P, Komarowska H, Czarnywojtek A, Waligórska-Stachura J, Bączyk M, Ziemnicka K, Fischbach J, Wrotkowska E, Ruchała M. Familial syndromes associated with neuroendocrine tumours. Contemp Oncol (Pozn) 2015; 19: 176-183.

2. Bilezikian JP. Primary Hyperparathyroidism. 2012 Feb 28. In: De Groot LJ, Beck-Peccoz P, Chrousos G, Dungan K, Grossman A, Hershman JM, Koch C, McLachlan R, New M, Rebar R, Singer F, Vinik A, Weickert MO (eds.). Endotext [Internet]. South Dartmouth (MA): MDText.com, Inc.; 2000. Available at: http://www.ncbi.nlm.nih. gov/books/NBK278923/.

3. Thakker RV. Multiple endocrine neoplasia type 1 (MEN1) and type 4 (MEN4). Mol Cell Endocrinol 2014; 386: 2-15.

4. Brandi ML, Gagel RF, Angeli A, et al. Guidelines for diagnosis and therapy of MEN type 1 and type 2. J Clin Endocrinol Metab 2001; 86: 5658-71.

5. Gut P, Fischbach J, Kamiński G, Ruchała M. Contemporary methods of therapy and follow-up of neuroendocrine tumours of the gastrointestinal tract and the pancreas. Contemp Oncol (Pozn) 2012; 16: 371-5.

6. Lassen T, Friis-Hansen L, Rasmussen AK, Knigge U, Feldt-Rasmussen U. Primary hyperparathyroidism in young people. When should we perform genetic testing for multiple endocrine neoplasia 1 (MEN-1)? J Clin Endocrinol Metab 2014; 99: 3983-7.

7. Simon K, Serafińska S, Pazgan-Simon M. Surveillance programmes for early detection of hepatocellular carcinoma. Contemp Oncol (Pozn) 2012; 16: 295-9.

8. Świątkowski M, Meder A, Sobczyński L, Koza J, Szamocka M, Brudny J, Korenkiewicz J. Adenomas detected during screening colonoscopies in the years 2000-2009. Prz Gastroenterol 2012; 7: 299-305.

9. Świątkowski M, Meder A, Sobczyński L, Koza J, Szamocka M, Brudny J, Korenkiewicz J. Serrated polyps detected during screening colonoscopies. Contemp Oncol (Pozn) 2014; 18: 54-9.

\section{Address for correspondence:}

\section{Jarosław Koza MD, PhD}

Chair of Gastroenterology and Nutrition Disorders Collegium Medicum Nicolaus Copernicus University Ujejskiego 75

85-168 Bydgoszcz, Poland

phone/fax: +48 523714912

e-mail: jaroslaw.koza@cm.umk.pl 